\title{
Perspective of computational modeling of nanomaterials
}

\section{Nordlund, K.}

Elsevier Ltd.

2020

Nordlund, K 2020 , Perspective of computational modeling of nanomaterials . in P

Grammatikopoulos (ed.), Computational Modelling of Nanomaterials . vol. 17 , Frontiers of

Nanoscience , vol. 17 , Elsevier Ltd. , pp. 1-3 . https://doi.org/10.1016/B978-0-12-821495-4.00001-4

http://hdl.handle.net/10138/336454

https://doi.org/10.1016/B978-0-12-821495-4.00001-4

cc_by_nc_nd

acceptedVersion

Downloaded from Helda, University of Helsinki institutional repository.

This is an electronic reprint of the original article.

This reprint may differ from the original in pagination and typographic detail.

Please cite the original version. 


\title{
Perspective of computational modelling of nanomaterials
}

\author{
K. Nordlund* \\ Department of Physics, P. O. Box 43, \\ FIN-00014 University of Helsinki, Finland
}

(Dated: November 19, 2019) 


\section{HISTORY}

Nanotechnology and nanoscience is commonly considered a new activity, and the use of the terms indeed is, as it clearly became popular after the inspiring, yet somewhat controversial book by Drexler [1]. However, it is good to recognize that nanoparticles have existed and even been used for practical applications long back in history. Nanoparticles form daily in copious amounts in nature as a byproduct of photosynthesis in common plants [2]. Burning of regular wood or a candle makes carbon (soot) nanoparticles, Romans used metal nanoparticles to get beautiful optical effects in glass [3], and the origin of the strength of the original Damascene swords lies in metal impurity nanoparticles [4].

In those ancient times, there was naturally no knowledge that the effects came out of the nanometer scale. Scientific understanding of the nanoscale can only have emerged after the development of chemistry, determination that atoms are physical discrete objects with sizes of the order of $0.1 \mathrm{~nm}$ [5] consisting of a very small positive core surrounded by the electron shell [6]. Early scientific study at the nanometer scale (before the nanotechnology buzzword was taken into use) included e.g. the extensive fields of colloid chemistry and thin film studies. A prime example of nanoscience-relevant early results is when Pawlow in 1907 obtained the still highly relevant result that the melting point of small particles scales with 1/diameter [7].

\section{RISE OF NANOSCIENCE}

In addition to the possible inspiration of Drexlers book, several independent scientific developments in the 1970's and 1980's naturally increased the interest into the nanometer scale. The miniaturization of silicon technology predicted by Moore's law [8, 9] inevitably lead to interest in making nanoscale components, while the invention of atomic force microscopy and improvements in e.g. electron microscopy made the scale more accessible.

Currently, the scientific study of nanomaterials, is a huge field, see Fig. 1. This publication data shows that the field has grown enormously since around 1990, and can still thus be considered a relatively young field. The youth, excitement, and doubtless also the increased research funding 


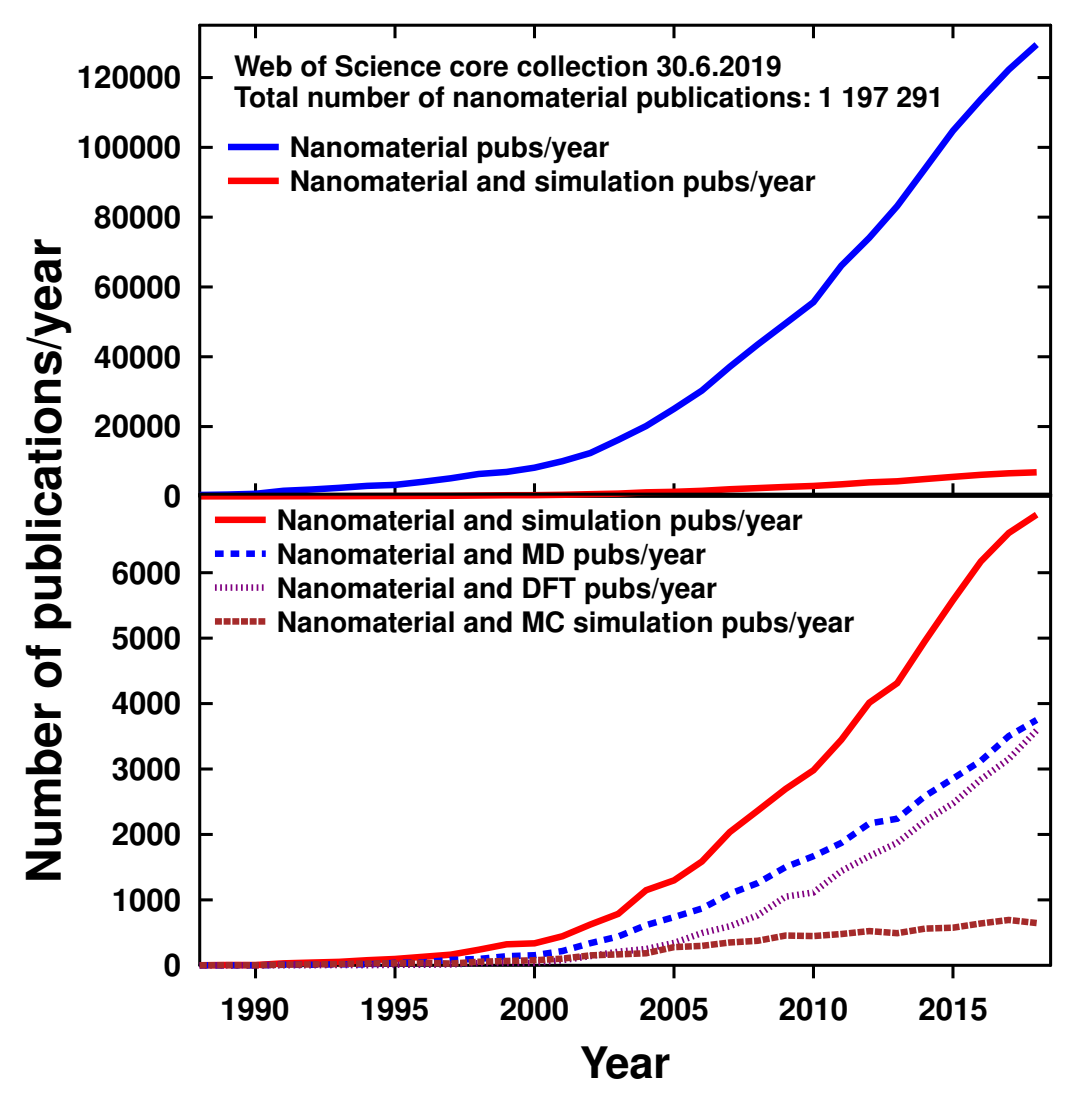

FIG. 1. Results of Web of Science Core Collection search on 30.6.2019 on "(nanoparticle* OR nanocluster* OR graphene OR nanowire* OR nanotube* OR nanocrystal* OR nanocrystalline OR "quantum well" OR "quantum dot")" in the publication topic (title or abstract). The lower curve and graph shows refinement of the search with they additional keyword "simulation*" or separately "molecular dynamics", "density functional theory" or "Monte Carlo". Note that the term "Monte Carlo" may refer to several completely distinct simulation methods.

to nanoscience, also leads to many scientists having entered the field from other, more traditional ones. For instance just for the 4subfield of studying individual nanoclusters in a vacuum or dilute gas (well represented in this book), it is easy to recognize several logical entryways to the field. One can enter from atomic and molecular physics, since studying small nanoclusters or fullerenes is in many ways a logical extension to small molecules. One can enter from ion beam physics, since setting up ion and cluster vacuum deposition equipment is in many ways similar. One can enter from macroscopic materials science, since study of small nanocrystalline materials is a logical extension of polycrystalline ones. One can enter from any molecular dynamics (MD) background, 
since studying nanoclusters in vacuum is conceptuallly simple in this method, and simulations of a small number of atoms very fast. Other logical entrance backgrounds are, for obvious reasons, surface physics and catalytic chemistry.

This variety of backgrounds clearly is a strength for the field, in that different backgrounds allow for different viewpoints in the studies of the nanoobjects. Atomic physicists will naturally know about discrete electronic orbitals, ion beam physicists about ion sources and acceleration, materials scientists about dislocations and grains, chemists about catalysis, and so on.

\section{COMPUTER SIMULATION OF NANOMATERIALS}

Nanoscience deals per definition with objects larger than a few atoms, but still smaller in number than those on the macroscopic scale, where thermodynamic averaging works well. Hence the physics of nanomaterials includes complex many-body interactions, which only very seldom are amenable to treatment by analytical models. Due to this, computer simulation methods are the preeminent tool for studying the nanoscale theoretically. Moreover, due to continued advances in computer capacity and algorithms, the capabilities for computer simulations will keep increasing, and hence also their importance for nanoscience.

The literature search results shown in Fig. 1 demonstrate that while most of the work in the field of nanomaterials is experimental, also the use of computer simulations for studying nanomaterials is a major endeavour. The total volume, from this simple literature search that certainly will miss many works, is about 7000 publications per year, which is a very respectable amount. To put this number in some perspective, by the Web of Science classifications the entire - including also experimental work - field "meteorology atmospheric sciences" is only about twice this size, and the field "biophysics" only thrice.

The search carried out shows that of the computational methods used, the dominant method is molecular dynamics (MD) $[10,11]$. The quantum mechanical method density functional theory (DFT) [12-14] is also in a rapid and comparable rise (in many cases both methods are used in the same papers or together, so there is certainly overlap in the data). The figure also shows that various Monte Carlo techniques such as Metropolis Monte Carlo [15] and Kinetic Monte Carlo 
[16-18] are also used, but their use is not in an equally rapid rise. On the other hand, the topic of using machine learning or artificial intelligence to study nanomaterials is currently raising very rapidly, from a volume of less than 20 publications/year before 2014, to more than 200 in 2019 . While this number is still much smaller than the other methods shown in the graphs, this broad class of advanced computation methods will doubtless continue to rise in research use and give significant additional insights in the field.

From these simple considerations, we see that the field of computational study of nanomaterials is clearly interesting and funding thousands of scientists, and attracting even more. The rapid development implies that the field is certainly not mature, but will keep evolving in the coming decades. Perhaps a bit paradoxically, the rapid evolution and large publication volumes means that it can be very worthwhile to "freeze a moment of time" in the field by gathering this book of top experts reviewing the current state-of-the-art in the field. The book can give an easier accessible overview of the field than all the thousands of individual articles, by highlighting selected recent key results and the current capabilities of the methods used. Thus it can be good reference of the state of the field in 2019 both for current and future generations of scientists.

* Corresponding authorkai.nordlund@helsinki.fi

[1] K. E. Drexler, Engines of Creation: The Coming Era of Nanotechnology (Doubleday, ADDRESS, 1986).

[2] P. M Winkler, G. Steiner, A. Vrtala, H. Vehkamäki, M. Noppel, K. E J Lehtinen, G. P Reischl, P. E Wagner, and M. Kulmala, Science (New York, N.Y.) 319, 1374 (2008).

[3] D. J. Barber and I. C. Freestone, Archaeometry 32, 33 (1990).

[4] J. D. Verhoeven, Scientific American 284, 62 (2001).

[5] A. Einstein, Ann. Phys. 17, 549 (1905).

[6] E. Rutherford, Phil. Mag. 6, 31 (1911).

[7] P. Z. Pawlow, Z. Phys. Chemie 65, 1 (1909), see also addendum, ibid. p. 545.

[8] G. Moore, Electronics 38, 4 (1965), original Moore's law publication.

[9] T. N. Theis and H. S. P. Wong, Computing in science \& engineering 19, 41 (2017).

[10] B. J. Alder and T. E. Wainwright, J. of Chem. Phys. 31, 459 (1959).

[11] M. P. Allen and D. J. Tildesley, Computer Simulation of Liquids (Oxford University Press, Oxford, 
England, 1989).

[12] P. Hohenberg and W. Kohn, Phys. Rev. 136, B864 (1964).

[13] W. Kohn and L. J. Sham, Phys. Rev. 140, A1133 (1965).

[14] R. O Jones, Rev. Mod. Phys. 87, 897 (2015).

[15] N. Metropolis, A. W. Rosenbluth, M. N. Rosenbluth, A. H. Teller, and E. Teller, J. Chem. Phys. 21, 1087 (1953).

[16] W. M. Young and E. W. Elcock, Proc. Phys. Soc 89, 735 (1966).

[17] A. B. Bortz, M. H. Kalos, and J. L. Lebowitz, J. Computational Physics 17, 10 (1975).

[18] K. A. Fichthorn and W. H. Weinberg, J. Chem. Phys 95, 1090 (1991). 